Revue d'histoire de l'enfance " irrégulière »

Le Temps de l'histoire

$11 \mid 2009$

Paroles libres, paroles captives

\title{
La quête des origines face à la loi du secret
}

Lettres d'enfants de l'Assistance publique (1900-1920)

\section{Antoine Rivière}

\section{CpenEdition}

Journals

Édition électronique

URL : http://journals.openedition.org/rhei/3060

DOI : $10.4000 /$ rhei.3060

ISBN : 978-2-7535-1650-2

ISSN : $1777-540 \mathrm{X}$

Éditeur

Presses universitaires de Rennes

\section{Édition imprimée}

Date de publication : 1 octobre 2009

ISBN : 978-2-7535-0927-6

ISSN : 1287-2431

Référence électronique

Antoine Rivière, "La quête des origines face à la loi du secret », Revue d'histoire de l'enfance « irrégulière » [En ligne], 11 | 2009, mis en ligne le 01 octobre 2011, consulté le 03 décembre 2020. URL : http:// journals.openedition.org/rhei/3060; DOI : https://doi.org/10.4000/rhei.3060

Ce document a été généré automatiquement le 3 décembre 2020.

(c) PUR 


\title{
La quête des origines face à la loi du secret
}

\author{
Lettres d'enfants de l'Assistance publique (1900-1920)
}

Antoine Rivière

1 De la fin du XIX siècle aux lendemains de la première guerre mondiale, entre 3000 et 4000 enfants sont abandonnés chaque année, dans plus de neuf cas sur dix par leur mère $e^{1}$, entre les mains de l'Assistance publique de Paris et placés sous sa tutelle. Devenus pupilles de l'État, ces enfants sont envoyés en province dans des familles nourricières, où, conformément à la doctrine traditionnelle de l'Assistance publique et à la loi de 1904 sur les enfants assistés, ils sont laissés dans l'ignorance de leur filiation. Comme l'ont montré les travaux historiques pionniers sur cette question ${ }^{2}$, qu'elle soit heureuse ou malheureuse, cette vie familiale de substitution ne parvient que rarement à détourner ces enfants de l'obsédante question de leurs origines. C'est ce que révèlent les dossiers individuels des enfants, dans lesquels l'administration consigne tout ce qui concerne ses pupilles jusqu'à leur majorité et parfois au-delà ; parmi 2200 dossiers d'enfants, qui, abandonnés à l'Assistance parisienne entre 1904 et 1923, ont survécu jusqu'à l'âge de l'apprentissage de la lecture et de l'écriture ${ }^{3}$, plus d'un sur quatre renferme les traces des questionnements existentiels qui assaillent ces jeunes sans famille: d'où viennent-ils? Pourquoi ont-ils été rejetés par ceux qui les ont mis au monde? Dans la correspondance que les pupilles nés entre 1900 et le début des années 1920 entretiennent avec le directeur de l'Assistance publique de Paris, leur tuteur officiel ${ }^{4}$, cette volonté de percer le mystère de leurs origines occupe donc une place importante, et détonne parmi les questions d'ordre pécuniaire, les demandes d'autorisation de mariage ou d'engagement militaire.

2 Face à une administration dont ils soupçonnent, parfois à tort, qu'elle sait tout de leur histoire personnelle, de l'identité de leurs parents et des motifs de leur abandon, ces jeunes déploient différentes stratégies épistolaires pour tenter d'infléchir la règle du secret. Quel que soit le ton choisi, celui de la confidence qui recherche l'empathie, ou celui plus péremptoire qui revendique le droit de connaitre ses origines, toutes ces lettres laissent transparaître la profonde souffrance de ne pas savoir d'où l'on vient ; 
souffrance que jusqu'au soir de leur vie, ces abandonnés portent comme une infirmité et que souvent, bien qu'atténuée, ils transmettent comme une nouvelle part de l'identité familiale à leurs descendants. Cette recherche de l'identité de leurs parents et des circonstances de la séparation, les pupilles l'entreprennent surtout à leur majorité, comme si la levée du secret devait marquer leur entrée dans l'âge adulte. Mais derrière ce qui est vécu comme un rituel de passage, ces écrits révèlent aussi le roman familial qu'ont pu construire ces jeunes pour remplacer tant bien que mal les pièces manquantes de leur histoire personnelle.

\section{La loi du secret}

3 Des débuts de la Troisième République jusqu'à la fin des années $1970^{5}$, l'administration oppose à ses pupilles et anciens pupilles une fin de non-recevoir systématique à leurs demandes de renseignements sur l'identité de leurs parents ou sur les conditions dans lesquelles ils ont été abandonnés. Par exemple, sur une cohorte de 72 pupilles admis en $1923^{6}$ et ayant fait des démarches pour connaître leurs origines, 20 obtiennent des renseignements ${ }^{7}$, soit un taux de réussite relativement élevé de $28 \%$; mais ce chiffre est trompeur, car aucune réponse favorable n'a été donnée par l'administration avant novembre 1980, et tous les anciens pupilles qui ont obtenu satisfaction au soir de leur vie avaient auparavant, souvent dès les années 1940, formulé des demandes identiques et essuyé des refus.

4 Dans le cas des enfants admis à l'Assistance publique de la Seine comme " trouvés " ${ }^{8}$, la question de la communication de ces renseignements ne se pose pas, car le propre de cette catégorie de pupilles est de réunir les enfants, dont l'administration ignore l'identité des parents. Qu'ils aient été exposés dans un lieu public ${ }^{9}$, que la personne qui les a déposés à l'hospice ait "refusé de se faire connaître ${ }^{10}$ ou qu'ils aient été inscrits à l'état civil avec la mention "né de père et de mère non dénommés ", ces enfants ne peuvent espérer recevoir de l'administration aucune réponse aux questions qu'ils se posent sur leurs origines ${ }^{11}$. En revanche, les dossiers des pupilles immatriculés comme "abandonnés » renferment des informations souvent précises sur les circonstances de leur arrivée à l'hospice, leur lieu de naissance ou l'identité de leurs père et mère. Hormis quelques cas, où le secret est explicitement demandé par la mère lors de l'abandon, le silence de l'administration repose sur des bases légales très générales, puisqu'il se fonde alors uniquement sur l'article 36 de la loi du 27 juin 1904 qui dispose : «L'article $378 \mathrm{du}$ code pénal, relatif au secret professionnel, est applicable à toute personne engagée dans le service des enfants assistés. [...] Dans tous les cas où la loi ou des règlements exigent la production de l'acte de naissance, il pourra y être suppléé si le préfet estime qu'il y a lieu d'observer le secret, par un certificat d'origine, dressé par l'inspecteur et visé par le préfet ». Quant à la possibilité - toute théorique - de déroger au principe de la non-communication aux pupilles des renseignements relatifs à leurs parents naturels, elle reste du seul ressort du tuteur légal, le directeur de l'Assistance publique de Paris, dont toutes les décisions doivent se fonder, dit l'exposé des motifs de la loi de 1904, sur un unique critère, dont l'interprétation est laissée à l'administration : «l'intérêt de l'enfant".

5 Malgré la place discrète que les textes législatifs et réglementaires lui font, le secret de la filiation des enfants est bien au cœur de la politique menée par les pouvoirs publics en matière d'abandon. En premier lieu parce que, parallèlement à sa mission 
d'assistance qui s'affirme avec une vigueur nouvelle au début de la Troisième République, le service des enfants assistés continue d'assumer sa vocation première: préserver l'ordre public en évitant qu'une femme, «fille-mère » ou épouse adultère, désireuse de cacher une naissance, dont la révélation risquerait de ruiner sa réputation et d'entacher l'honneur de sa famille, ne se résolve à avorter ou à commettre un infanticide. Sur ce point, la doctrine de l'Assistance publique de la Seine, confirmée par la loi du 27 juin 1904, est solidement ancrée depuis les années 1880 : toute personne se présentant à l'hospice départemental pour y faire un abandon doit recevoir l'assurance qu'aucune indiscrétion de la part de l'administration ne révélera jamais son identité, pas même à l'enfant qu'elle a déposé. Garantir le secret des origines des enfants abandonnés constitue bien, comme le dit Paul Strauss ${ }^{12}$, ancien responsable du service des enfants assistés de la Seine et figure majeure des politiques de protection maternelle et infantile sous la Troisième République, un « moyen de prévenir les crimes contre l'enfance $»^{13}$. Mais cette politique du secret sert d'autres fins; et la position de principe de l'Assistance publique, consistant à ne divulguer à ses pupilles aucun renseignement sur leurs parents ou sur les circonstances de leur abandon, vise aussi à éviter qu'ils ne parviennent, sans l'accord explicite de l'administration, à retrouver leurs parents naturels. La grande crainte des pouvoirs publics est en effet que des retrouvailles trop faciles n'encouragent les parents les plus pauvres à considérer l'abandon comme une simple mise en pension temporaire aux frais de l'État, le temps que l'enfant ne soit plus seulement une bouche à nourrir mais aussi une force de travail. Le secret de la filiation, qui permet à l'administration de contrôler le retour des ex-pupilles dans leur milieu d'origine, est donc conçu comme une nécessité pour les finances du service, en même temps qu'il est pensé comme un moyen de responsabilisation et de moralisation des familles populaires qui doivent assumer l'éducation et l'entretien de leurs enfants, sans espérer s'en décharger sur la collectivité. Reste que l'Assistance publique conçoit surtout cette rupture avec la famille d'origine comme la condition sine qua non de la réalisation de son œuvre de redressement moral des enfants qui lui sont confiés. Car, comme l'a démontré Ivan Jablonk $\mathrm{a}^{14}$, son projet éducatif porte haut une ambition régénératrice, qui consiste à inculquer aux pupilles des valeurs et des mœurs nourries de l'idéologie positiviste et agrarienne de la jeune république et largement ignorées de parents dont l'appartenance au prolétariat urbain le plus misérable, la "vie de débauche", l'alcoolisme ou la maladie - syphilis ou tuberculose, par exemple - signent aux yeux de l'administration leur incapacité à éduquer correctement leur progéniture. Permettre aux pupilles de renouer avec leur milieu d'origine risquerait de les rendre à l'influence corruptrice de parents détruits, aussi bien physiquement que moralement, par l'usine et l'assommoir; ce serait réduire à néant les efforts, supposés bienveillants et bénéfiques, des nourriciers et des personnels de l'Assistance publique.

6 Face à cette culture du secret, aucun homme politique, aucun réformateur social, aucune œuvre privée de protection de l'enfance ne conçoit à l'époque un quelconque droit des pupilles à connaître leurs origines. Parviennent tout juste à se faire entendre quelques voix qui s'émeuvent de ce que l'État républicain, garant de l'état des personnes et des droits découlant de "l'acte civil de naissance, [...] une des plus précieuses conquêtes de la Révolution $»^{15}$, se rende complice de pratiques attentatoires aux droits individuels des pupilles, notamment en matière d'héritage, en créant la possibilité de l'abandon anonyme et en organisant une stricte rétention des informations relatives à leur filiation. Mais ces scrupules pèsent bien peu face à 
l'argumentaire bien rôdé des populationnistes : «mieux vaut une suppression d'état qu'une suppression d'enfant $»^{16}$. La cause est entendue ; et, témoignant pour tous les enfants abandonnés, un ancien pupille, admis en 1918 à l'Assistance publique de la Seine, peut, au soir de sa vie, tirer l'amère leçon des refus catégoriques que l'administration a opposés à toutes ses demandes de renseignements sur l'identité de sa mère : «"Secret" ! J'étais au secret ! ${ }^{17}$.

\section{À l'orée de la vie d'adulte : la volonté de savoir}

7 Cette culture du secret, partagée par tous les personnels du service des enfants assistés, est tellement prégnante que c'est parfois avec le sentiment de transgresser un interdit que certains enfants s'enquièrent de leurs origines auprès de leur directeur d'agence, de l'inspecteur des enfants assistés ou du directeur de l'Assistance publique; comme cet ancien pupille de la Seine, qui se souvient : «il était impérativement déconseillé d'entreprendre des recherches $\aleph^{18}$. À mesure qu'ils grandissent et qu'ils voient se rapprocher le moment de leur émancipation, cet obstacle parait cependant de moins en moins insurmontable, et les pupilles ne s'embarrassent pas de savoir si leur démarche déplait à ceux qui, du nourricier au tuteur, régentent leur vie. Car, heureux ou malheureux dans leur placement ${ }^{19}$, qu'ils aient été délaissés à la naissance ou quelques années plus tard, ils sont, pour une grande partie d'entre eux, obsédés par les zones d'ombre de leur histoire personnelle, et ne peuvent accepter que les questions essentielles qu'ils se posent restent sans réponses : qui sont leurs parents? Pourquoi les ont-t-ils abandonnés?

8 Lorsqu'approche l'âge de la majorité, ce désir de savoir semble se faire particulièrement pressant. Paradoxalement, alors qu'ils sont conscients que la question de leur filiation est un tabou entretenu avec constance par l'institution, bon nombre de ces pupilles semblent vivre leurs jeunes années avec l'idée, parfaitement irrationnelle, que la fin de leur minorité sera inaugurée par la révélation de leurs origines ${ }^{20}$. Ils attendent donc ce moment avec impatience, voire, pour certains qui s'apprêtent à fêter leurs vingt et un ans, avec une fébrilité certaine; car s'ils ne doutent pas que l'administration connaisse l'identité de leurs père et mère, ils entretiennent aussi l'espoir que le silence sera brisé lorsqu'ils entreront eux-mêmes dans cette communauté des adultes, qui savent tout et qui, jusque-là, ne disent rien. Tout se passe en effet comme si, à leurs yeux, devenir majeur c'était être reconnu par l'administration comme des individus véritablement responsables, capables d'affronter l'histoire de leur abandon et aptes à juger par eux-mêmes de l'opportunité de renouer avec cette mère qui les a rejetés. En ce sens, l'écriture de la lettre, que le pupille adresse à son tuteur pour connaître sa filiation et les motifs de son abandon, est sans doute déjà vécue comme un premier rituel, censé préparer l'entrée dans l'âge adulte. Que la levée du secret accompagne leur émancipation de la tutelle de l'institution semble donc parfois aller de soi dans l'esprit de certains pupilles ${ }^{21}$; et ce jeune homme, avec quelque ingénuité, n'imagine pas qu'il puisse en être autrement, lorsqu'il écrit en mars 1937 : "Je voudrais connaître quelques précisions sur l'identité de ma famille, vu que j'ai eu 21 ans le mois de décembre dernier ${ }^{22}$. Lorsque l'administration répond à la requête de ses pupilles en affirmant que leur dossier renferme uniquement des renseignements très vagues, qui de toutes façons ne leur sont pas communicables, leurs attentes sont donc doublement déçues. D'une part, ils sont incrédules et se sentent trahis par leur 
tuteur, comme ce pupille qui, après des échecs répétés auprès de l'hospice de la rue Denfert-Rochereau, du directeur d'agence, puis de la direction de l'Assistance publique, décide de s'adresser au ministre de la justice : «je ne dois pas certainement être seul, il y a bien une mère qui m'a fait [...] veuillez bien faire votre possible pour me donner des renseignements que vous pouvez posséder certainement. ${ }^{23}$ D'autre part, ils vivent la prolongation du silence administratif comme l'aveu douloureux qu'ils ont démérité, qu'ils ne sont pas jugés dignes ni suffisamment capables de connaître leur passé; d'autant que certains indices témoignent de ce que l'administration elle-même semble donner foi à l'hypothèse d'un régime de faveur, qui pourrait être accordé à des pupilles particulièrement méritants : certes les circonstances sont exceptionnelles, mais c'est bien cette idée que caresse le service des enfants assistés de la Seine, lorsque dans son rapport de l'année 1918, il évoque, à propos des pupilles mobilisés tout au long de la Grande Guerre, des « jeunes gens qui invoquent leur glorieuse conduite pour solliciter une faveur qu'ils semblent avoir bien méritée ${ }^{24}$ : celle de connaître leur mère. $\aleph^{25}$ Plus généralement, l'attente des pupilles est sans doute entretenue par le modèle de la récompense qui, entre autres, régit les rapports entre l'institution et les enfants ${ }^{26}$ : l'administration incite à la bonne conduite en distribuant aux plus sages des jouets ou des livres, puis, lorsque les pupilles grandissent, les plus travailleurs, les plus méritants reçoivent gratifications en argent et autres "primes de sagesse ${ }^{27}$. Dans l'esprit de quelques-uns de ces jeunes gens, il semble que la récompense ultime de leur comportement modèle devrait être la levée du secret de leurs origines.

9 La déception de ces pupilles qui, à l'orée de leur vie adulte, s'enquièrent de leurs parents biologiques, est d'autant plus grande que, croient-ils savoir, «il y a déjà bien des camarades qui ont trouvé leur famille $»^{28}$. Une telle certitude est vraisemblablement entretenue par la différence de traitement entre, d'une part, les abandonnés et les trouvés, et, d'autres part, les enfants d'autres catégories, comme les orphelins, les recueillis temporaires ou les moralement abandonnés, dont la filiation n'est soumise à aucun secret. Mais le sentiment d'injustice qui en découle est encore renforcé par le fait que l'administration autorise certains parents qui en font la demande à renouer avec l'enfant qu'ils ont abandonné. Outre les «remises» de pupilles, les «mises en relations $»^{29}$ sont accordées au terme d'une longue procédure, qui vise notamment à vérifier par une enquête minutieuse que les parents sont respectables, guidés par des motivations désintéressées et qu'ils ont régulièrement pris des nouvelles de l'enfant ${ }^{30}$. Subordonnées au consentement des pupilles, elles ne concernent que des jeunes proches de la majorité, et entretiennent chez les autres abandonnés l'espoir de possibles retrouvailles au moment de l'émancipation. Bien que peu nombreuses ${ }^{31}$, elles incitent donc les pupilles à tenter leur chance auprès de l'administration, quitte à rendre plus douloureuse encore la déception de ceux qui se heurtent à un refus, parce que leurs parents se sont définitivement détournés d'eux.

Outre leur vingt et unième anniversaire, les étapes importantes qui marquent généralement leur vie de jeunes adultes sont autant d'occasions pour les anciens pupilles de s'enquérir de leurs ascendants. Le mariage, qui les fait entrer dans une nouvelle famille et leur ouvre la perspective d'en prolonger l'arbre généalogique, ravive souvent leur questionnement sur leur propre filiation. Quant à la naissance prochaine d'un enfant, elle donne souvent lieu, elle aussi, à de nouvelles tentatives pour percer à jour le secret des origines. En 1946, un jeune employé de la SNCF, abandonné à la naissance en 1916, écrit à son ancien tuteur : 
«Marié depuis l'année dernière, je suis appelé à avoir des enfants, je l'espère d'ailleurs, et c'est cette idée, Monsieur le Directeur, qui me donne des inquiétudes, car je ne sais pas du tout quelle hérédité ils peuvent avoir. Aussi, je vous serais infiniment reconnaissant s'il vous était possible de m'éclairer un peu sur ce sujet. ${ }^{32}$

11 Cette lettre montre une partie des angoisses auxquelles peuvent être confrontés d'anciens pupilles, ignorants de leurs origines, qui s'apprêtent à fonder une famille et qui s'interrogent sur ce qu'ils transmettront à leur descendance : tares héréditaires, traits de caractère familiaux ou troubles affectifs dus au traumatisme psychologique de l'abandon. Loin d'être le fait d'un stratagème inventé par quelques jeunes gens astucieux, cette inquiétude des pupilles au sujet de ce qu'ils ont pu hériter des caractères de leurs parents renvoie, semble-t-il, à une véritable préoccupation de l'époque, entretenue par certaines avancées scientifiques ${ }^{33}$, et trouve parfois un écho auprès de leurs proches. Une ancienne pupille âgée de 27 ans, qui doit épouser un soldat américain, explique, dans une lettre adressée en 1947 au directeur de l'Assistance, les réticences de ses futurs beaux-parents à marier leur fils à une femme dont ils ne connaissent rien de l'ascendance :

«[...] je voudrais connaitre la raison sous laquelle je suis été abandonnée et si j’ai encore des parents. Monsieur, je suis fiancée depuis trois ans et ne connaissant pas mes parents, mon fiancé hésite à se marier avec moi et surtout sa famille. Il ne suffit donc pas de souffrir étant enfant de ne pas avoir ni père ni mère comme tout le monde mais toute sa vie il faut supporter la faute de parents indignes. $»^{34}$

Qu'elle serve aux parents du fiancé de prétexte pour repousser une union qu'ils n'approuvent pas, ou qu'elle alimente une véritable inquiétude au sujet d'une famille qu'ils imaginent peu respectable, voire dégénérée, l'ignorance de l'ascendance de l'ancienne pupille apparaît comme un véritable handicap ; pour sa part, la jeune fille la vit comme une malédiction dont, sans ambiguïté, elle rend ses père et mère entièrement responsables.

13 Parmi les rites qui jalonnent le passage à l'âge adulte, il en est un, spécifique aux garçons, qui apparait particulièrement propice à ces tentatives épistolaires d'infléchir la stricte règle du secret prônée par l'Assistance publique : le service militaire. Pour la plupart de ces jeunes hommes, le départ à l'armée est en effet la première occasion qui leur est donnée de quitter l'univers quotidien des enfants de l'Assistance, et d'échapper, au moins pour un temps, à un milieu, qui, du nourricier au directeur d'agence, "déconseille d'entreprendre des recherches $»^{35}$; il leur est alors loisible d'écrire à leur tuteur, d'autant plus que la vie de garnison les laisse souvent désœuvrés. Sous les drapeaux, les pupilles font surtout l'épreuve décisive de leur grande solitude, et, au milieu de camarades de régiment qui reçoivent le réconfort de lettres ou de colis envoyés par les parents, ils ressentent cruellement l'anormalité de leur condition d'enfants sans famille. Dans le cas particulier des pupilles mobilisés au début de la seconde guerre mondiale, puis faits prisonniers et envoyés en Allemagne, le même sentiment d'extrême solitude, aggravé par les conditions et la durée de la détention, transparaît dans les lettres adressées à l'administration. Du camp de prisonniers de guerre situé en Westphalie, où il est enfermé depuis juin 1940, un ancien pupille écrit ainsi, en février 1942 : "Je serais heureux de trouver de la famille. Je suis prisonnier depuis 21 mois, je voudrais bien sortir, c'est une drôle de vie. $»^{36}$ Au-delà des humiliations, du froid, des privations alimentaires ou de la pénibilité du travail forcé, ce jeune homme souffre d'un isolement qui, à la différence de celui qu'endurent ses 
compagnons de captivité, n'est pas même rompu par quelques lettres attentionnées d'un père ou d'une mère inquiets de son sort. La plupart de ceux qui ont vécu la guerre et l'enfermement, parfois avec le sentiment qu'ils n'en reviendraient peut-être pas, puis qui ont éprouvé un grand désarroi lorsque, de retour en France, ils savaient qu'aucun parent ne les y attendait, ressortent de cette difficile expérience avec l'impérieux désir, mûri tout au long de leur captivité, de retrouver leurs parents. C'est ce cheminement douloureux qu'a suivi un pupille abandonné en 1919 à l'âge de sept mois; alors qu'auparavant il n'avait jamais sollicité l'administration au sujet de l'identité de ses parents, il écrit en mars 1946 au directeur de l'Assistance publique de Paris :

«Je viens auprès de vous pour savoir l'origine de ma naissance. Je rentre de captivité et pendant ces cinq années j'ai souvent pensé à mes parents, aussi, Monsieur, je vous serais reconnaissant si vous pouviez me donner un renseignement. ${ }^{37}$

Contrairement à d'autres, qui, au lendemain de la Grande Guerre, victorieux, auréolés de leur courageux esprit de sacrifice, arguaient de leur tout nouveau statut d'ancien combattant pour demander à l'administration qu'elle dissipe le mystère de leur naissance et de leur abandon, ce jeune homme, vaincu, prisonnier, ne saurait faire valoir aucune conduite héroïque ; tout au plus, à lire entre les lignes, peut-on imaginer qu'il laisse entendre au destinataire de la lettre que les souffrances endurées méritent bien une compensation. Quelles que soient les circonstances dans lesquelles pupilles et anciens pupilles se lancent à la recherche de leurs origines, leurs écrits, sous une apparente simplicité, démontrent en effet qu'ils savent mettre en œuvre toute une panoplie d'arguments, certains peut-être maladroits, d'autres subtiles, pour tenter de parvenir à leurs fins.

\section{Stratégies épistolaires}

$15 \mathrm{Au}$ cours de leur vie, les anciens pupilles envisagent plusieurs moyens de percer le mystère de leur histoire personnelle. Celui-là avoue au directeur : «j'ai pensé passer outre [les refus de l'administration] et faire appel aux compétences d'un détective privé $»^{38}$; celle-ci, au début des années 1990, interroge l'aide sociale à l'enfance : «je voudrais connaître tous les éléments de mon dossier, [...] est-ce possible? ou dois-je avoir recours à l'émission de la télé "Perdu de vue" ? »" ${ }^{39}$ De fait, certaines démarches officieuses, voire illégales, portent leurs fruits, notamment lorsque les anciens pupilles bénéficient de l'aide d'un proche, employé dans une administration publique ${ }^{40}$. Il semble cependant qu'ils n'empruntent ces voies parallèles qu'en désespoir de cause, car, spontanément, c'est vers leur tuteur qu'ils se tournent, dont ils espèrent des réponses, mais aussi une reconnaissance en quelque sorte officielle de leurs souffrances, un geste d'humanité et de compassion, et qu'ils pensent convaincre par des stratégies épistolaires souvent ingénieuses et d'une grande variété.

Voués à des professions manuelles, ouvrières ou plus souvent agricoles, les enfants poursuivent rarement leur scolarité au-delà de l'âge de treize $a^{41} s^{41}$ mais l'Assistance publique, porteuse de la mystique républicaine, notamment de la foi en l'instruction, veille scrupuleusement à ce qu'ils fréquentent régulièrement les bancs de l'école et apprennent au moins à lire, à écrire et à compter. S'ils ne sont pas toujours familiers de l'écrit, ils sont donc tous capables d'entretenir avec l'administration la correspondance 
que nécessite leur condition de pupilles de l'Assistance. Lorsqu'ils sont encore sous la tutelle de l'institution, ils doivent ainsi adresser une demande écrite au directeur chaque fois qu'ils veulent prélever de l'argent sur leur livret d'épargne et faire usage de leurs " deniers pupillaires "; lorsqu'ils entrent dans la vie adulte, il leur faut demander l'autorisation de leur tuteur pour se marier ou s'engager dans l'armée, et, dans toutes les occasions où les citoyens ordinaires doivent justifier, par un extrait de naissance, de leur identité et de leur filiation - mariage, succession, acquisition d'un bien immobilier ${ }^{42}$-, les anciens pupilles doivent, eux, écrire à l'Assistance publique, afin qu'elle leur fournisse le certificat d'origine qui leur tient lieu d'acte d'état civil. Il arrive aussi que les pupilles écrivent à l'administration au moment de leur entrée dans la vie active, afin de solliciter une aide à leur installation, notamment lorsqu'ils envisagent d'acheter un lopin de terre pour y développer une activité agricole. Enfin, bien que peu fréquentes dans les dossiers, quelques lettres adressées au directeur de l'Assistance publique ou au directeur d'agence n'ont d'autre ressort que la relation affective qui a pu se tisser entre le pupille et les responsables de l'institution; elles sont simplement écrites pour donner des nouvelles, faire part de la naissance d'un enfant, par exemple, ou annoncer le retour en métropole après un long service militaire dans les colonies. $\mathrm{Au}$ milieu de cette correspondance, qui, sans être abondante, est donc relativement courante, les écrits dans lesquels les pupilles interrogent l'administration sur leur histoire personnelle se démarquent: non seulement les individus s'y racontent beaucoup plus que dans les autres courriers, en dévoilant leurs espérances, leurs angoisses ou leurs souffrances intimes, mais ces lettres sont aussi plus complexes, plus travaillées et souvent plus longues, puisqu'il s'agit pour leurs auteurs de convaincre et de fléchir une administration qui a fait du silence des origines l'une de ses règles fondamentales.

17 En juin 1939, une ancienne pupille tout juste majeure, écrit au directeur de l'administration, qui, quelques semaines plus tôt, a refusé d'accéder à sa première demande :

«Je suis bien désolée de ne rien savoir de ma famille, vivre et ignorer sa propre origine cela me semble bien dur. Enfin, j'ai maintenant 21 ans, j'ai gagné ma vie du mieux que j'ai pu, je veux donc avoir le courage de continuer. $»^{43}$

18 Derrière les mots choisis par cette jeune femme, se déploie la stratégie la plus simple utilisée par les pupilles en quête de l'identité de leurs géniteurs. Elle consiste à rechercher l'empathie du tuteur, à susciter sa pitié, en lui dévoilant, parfois sur le ton de la confidence, l'intime souffrance qu'endure celui qui ignore tout de ses origines. Apparemment triviale, cette stratégie est en fait plus complexe qu'il n'y paraît, puisque elle invite souvent le destinataire à mettre en regard cette condition malheureuse d'enfant abandonné et la conduite irréprochable d'un pupille qui prétend avoir su tirer tout le profit de l'éducation que l'Assistance publique lui a dispensée. Implicitement, la question que posent les écrits de ce type est donc double : ce pupille exemplaire n'a-t-il pas mérité que le secret soit levé ? Si un tel pupille, honnête, travailleur, obéissant, n'est pas gratifié par la seule récompense qui vaille à ses yeux, à quoi bon être un pupille honnête, travailleur et obéissant? Il est même possible, semble-t-il, de voir derrière les mots de la jeune femme évoquée plus haut un chantage implicite: lorsqu'elle dit qu'elle espère trouver, malgré son désarroi, le courage de continuer à gagner sa vie du mieux possible, elle laisse entendre dans le même temps qu'elle pourrait ne pas y parvenir ; autrement dit, elle sous-entend que, laissée à elle-même puisque, comme elle le précise dans cette même phrase, elle est désormais émancipée 
de la tutelle de l'Assistance publique - elle pourrait tomber dans la misère ou, ce qui serait pire aux yeux de l'administration, mener une vie malhonnête, faite de crimes et de débauche ${ }^{44}$, qui signerait la faillite de l'éducation qu'elle a reçue du service des enfants assistés.

D'autres lettres reposent sur des ressorts plus complexes et peut-être moins brutaux; avec de nombreuses variantes, elles affirment toutes que si l'ancien pupille désire obtenir quelques renseignements sur ses parents biologiques, c'est uniquement pour assouvir sa curiosité généalogique ou, à la rigueur, pour calmer ses inquiétudes en matière d'hérédité, mais en aucun cas dans le but de renouer avec eux. L'ancien pupille devenu employé de la SNCF qui, envisageant sa paternité prochaine, s'inquiétait de son hérédité, écrit dans cette lettre de 1946, déjà citée :

«Jusqu'à aujourd'hui je ne me suis nullement intéressé de mon origine. J'ai eu le bonheur d'être recueilli dans une famille qui m'a toujours considéré absolument comme faisant partie des leurs [sic]. [...] Je vous serais infiniment reconnaissant s'il vous était possible de m'éclairer un peu sur [mes parents]. Notez, Monsieur le Directeur, que je ne veux en aucune façon, le cas échéant, entrer en relations avec des personnes que je méprise, je n'ai qu'une famille, celle qui m'a élevé [...]. »45

Qu'il soit ou non de bonne foi lorsqu'il nie vouloir retrouver ces parents qu'il méprise, ce jeune homme démontre dans ce texte la capacité des pupilles à intégrer parfaitement quelques-uns des topoï les plus saillants de l'ambition éducative de l'Assistance publique et à les mettre au service de leur propre volonté de connaître leurs origines. Tout l'art de ces écrits de la dénégation, consiste, stratégie et sincérité sans doute confusément et inextricablement mêlées, à donner à l'administration des gages de ce que son projet régénérateur de rupture avec la famille d'origine a porté ses fruits; les jeunes auteurs de ces lettres y affirment donc qu'ils sont absolument heureux de leur situation, très attachés à des nourriciers qui sont leur véritable famille et qu'en aucun cas ils ne souhaiteraient renouer avec un milieu certainement amoral, misérable et corrupteur, auquel ils se félicitent d'avoir échappé. Si le témoignage de la réussite éducative de l'administration peut ainsi servir, pensent-ils, la quête de leur histoire personnelle, c'est parfois a posteriori que les pupilles manifestent leur allégeance au projet et aux discours de l'Assistance, et minimisent leur intérêt pour leur famille d'origine. C'est le cas de cette jeune femme de 19 ans, qui après avoir demandé, au début de 1942, des renseignements sur les circonstances de sa "venue aux enfants assistés ", apprend de l'administration qu'elle a un frère, abandonné un an avant elle ; dans une nouvelle lettre, datée de juillet 1942, elle écrit :

«Monsieur le directeur, j'ai été très surprise en recevant votre lettre m'annonçant que j'avais un frère et me donnant son adresse. Je pensais n'avoir qu'une seule famille celle qui m'élève, mais comme ce jeune homme n'est pas cause du sort qui nous a été réservé, je vais lui écrire. Je pense que ça lui fera plaisir. Je suis très contente d'être infirmière c'est vraiment ce que je désirais, je vous remercie beaucoup de vous être occupé de moi [...]. $»^{46}$

Parfois, le récit de soi et la requête purement individuelle, laissent la place à une revendication plus collective sous la plume de ces jeunes qui commencent leur vie d'adultes. La préoccupation des origines est tellement courante lorsqu'arrive l'âge de la majorité, que les pupilles en conçoivent un fort sentiment d'appartenance à une même communauté, qui exclut ceux qui n'endurent pas les mêmes tourments qu'eux : «je ne sais si vous comprenez ce besoin de retrouver ses origines $"{ }^{47}$, écrit l'un d'eux au directeur, en 1980. Dans la correspondance de certains de ces jeunes gens, apparait alors l'expression, parfois confuse, souvent inédite, d'une identité collective, fondée sur 
la souffrance commune de ne pas savoir d'où l'on vient. En 1937, un pupille, qui va sur ses vingt et un ans, demande au directeur de l'Assistance publique des renseignements sur sa mère ; il écrit : « je voudrais qu'un effort soit fait pour moi comme pour ceux qui font comme moi, qui veulent savoir, car à notre âge c'est une pensée qui nous hante sans cesse $»^{48}$. Si c'est bien ce sentiment de partager avec les autres enfants abandonnés une même infirmité généalogique et affective qui conduit ce jeune homme à dépasser son seul cas personnel, sa démarche n'en demeure pas moins strictement individuelle. Cette prise de conscience d'une communauté de destin et de souffrance, qui devient véritablement visible dans l'entre-deux-guerres, peut-elle être considérée, malgré tout, comme le premier pas, encore timide, vers une forme d'action collective, organisée et proprement militante? Sans doute ; mais sur ce point, il faut suivre Ivan Jablonka : « la lutte des enfants assistés pour accéder à leurs origines est lente à se mettre en place ${ }^{49}$, puisqu'il faut attendre les années 1970 pour voir les anciens pupilles se mobiliser, au sein d'associations, sur le front de l'affirmation d'un droit à connaître leur filiation.

\section{Roman familial}

Dans la construction imaginaire qui apparaît en filigrane dans les lettres des pupilles, la mère tient une place prépondérante : c'est sur elle, bien plus que sur le père, que se focalisent les interrogations; c'est elle qui est l'objet de la plupart des récits fantasmatiques que s'inventent les enfants abandonnés. Plusieurs hypothèses peuvent l'expliquer. Il semble que les pupilles, comme les employés de l'Assistance publique, comme l'ensemble du corps social de l'époque, ont intégré l'idée que les parents abandonneurs sont majoritairement des mères seules, délaissées du père de l'enfant dès qu'elles lui ont appris leur grossesse. Ce dont témoigne cette ancienne pupille, abandonnée à la naissance en février 1923, dans une lettre qu'elle écrit à l'Aide sociale à l'enfance en 1980 : «Je ne parle pas de mon père car j'ai toujours pensé être née d'une fille-mère et que ma naissance de ce fait a été cachée, d'où cet abandon [...]. ॥ ${ }^{50}$ Le père est donc réputé n'avoir jamais porté aucun intérêt à sa progéniture et ne l'avoir même jamais connue, contrairement à la mère qui, au moins pendant les neuf mois de la grossesse, puis au moment de la naissance, a, elle, noué un lien avec son enfant. C'est sans doute l'existence de cette relation primitive, autant que le supposé instinct maternel auquel la société de l'époque, en définissant le rôle de chacun des genres, assigne les femmes, qui rendent particulièrement incompréhensible et douloureux pour les pupilles d'avoir été délaissés par leur mère, et alimentent à la fois leur questionnement sur les raisons de leur abandon, et les hypothèses qu'ils échafaudent pour y répondre.

Les écrits de ces jeunes en quête de renseignements sur leur filiation, révèlent en effet le roman familial qu'ils ont pu se construire pour apaiser la blessure de l'abandon et rendre un peu moins insupportable l'absence de ceux qui les ont mis au monde. Quelques-uns demandent seulement à lire les lettres que n'aura pas manqué d'écrire leur mère pour prendre de leurs nouvelles ou expliquer son geste. D'autres, à l'imagination plus débridée, cherchent des signes tangibles qui viendraient étayer le récit de leurs origines qu'ils ont fabriqué de toutes pièces. Tous, au fond, ne demandent finalement à l'administration qu'une confirmation de cette fiction qu'ils se sont inventée. Dans la plupart des cas, ces constructions fantasmatiques dédouanent la mère de la responsabilité de la séparation et lui prêtent un amour maternel sincère, 
seulement contrarié par des circonstances indépendantes de sa volonté. Cette hypothèse d'un abandon dû à des motifs extérieurs est parfois entretenue par des indices ténus; un pupille qui vient d'avoir ses vingt-et-un ans, écrit en 1936 au directeur de l'Assistance publique :

« Ces deux mots [...] pour vous demander à vouloir bien me mettre au courant si j'ai de la famille ou si je possède des parents [...]. Cela m'intéresse beaucoup, parce que ce qui m'étonne c'est que, ayant vu sur mon livre de pupille que mes parents m'avaient gardé près de 2 ans, c'est ce que je me décide à vous demander cela. $»^{51}$

24 À travers cette lettre, il apparaît que ce jeune homme, à partir de la seule information dont il disposait sur les conditions de son dépôt à l'hospice, a pu échafauder différentes hypothèses; l'ébauche de roman familial qu'il en a conçue tient dans cette question sous-jacente à son texte : si son père et sa mère n'étaient pas attachés à lui, pourquoi auraient-ils attendu deux ans avant de le confier à l'Assistance publique ? et le laisse espérer que ses parents ont pu ressentir pour lui une véritable affection et qu'ils n'ont accompli cet abandon tardif que sous l'influence de mystérieuses circonstances, étrangères à leurs sentiments pour sa personne. Certains pupilles, comme pour conjurer la douloureuse évidence que l'on n'a pas voulu d'eux, imaginent que leur mère s'est résolue à les abandonner, la mort dans l'âme, contrainte par la misère ou par une famille qui craignait pour son honneur et sa réputation. D'autres, à demi-mot, reprochent à l'Assistance publique de ne pas avoir répondu favorablement aux demandes répétées et insistantes de leur mère pour les reprendre auprès d'elle, et demandent des comptes à cette administration qui a fait leur malheur. L'agent de la $\mathrm{SNCF}$, ancien pupille de la Seine, que nous avons déjà rencontré, écrit dans sa lettre : "Avec des renseignements je pourrais quand même me rendre compte de certaines choses. J'aurais, parait-il, été recherché à l'âge de deux ans. $»^{52}$ Ses mots démontrent que, même ceux qui affichent un profond mépris pour leurs parents et qui nient tout désir de renouer avec eux, ne peuvent s'empêcher de se laisser aller à quelques inventions consolatrices; le dossier de ce pupille ne garde en effet aucune trace d'une quelconque demande de nouvelles, ni d'aucune démarche pour le reprendre. Ce que révèlent les écrits de ces jeunes c'est tout autant l'angoisse de ne pas connaître leurs origines que l'immense blessure narcissique d'avoir été délaissés par leurs parents ; car tous ces récits fictifs ont pour point commun de conforter leurs auteurs dans l'idée qu'ils ne sont pas responsables de leur propre abandon, qu'ils n'ont pas été rejetés parce qu'ils ne méritaient pas d'être aimés.

Ce roman familial tente aussi de combler un manque dans la construction identitaire de ces jeunes. Sans repères familiaux, sans exemples parentaux, malgré les efforts de nourriciers parfois aimants et dévoués, certains pupilles ressentent la nécessité de s'inventer des parents. Si cela transparaît rarement dans leur correspondance, le cas tout à fait particulier des pupilles masculins nés pendant la Grande Guerre met au jour ce processus. Au début du printemps 1940, alors qu'il est sous les drapeaux depuis octobre 1937, un ancien pupille, né en 1916 et abandonné à l'hospice des enfants assistés de la rue Denfert-Rochereau en 1919, écrit au directeur de l'Assistance publique de Paris :

« Ayant été abandonné à l'âge de trois ans, j'ai toujours cru que mon père avait été tué à la guerre [de 1914-1918], alors je viens vous demander si vous pourriez me donner des renseignements précis sur cette question le plus tôt possible, cela me rendrait peut-être grand service, étant susceptible de partir au front d'ici peu. ${ }^{53}$ 
ant pouvoir bénéficier des exemptions réservées aux enfants des soldats morts pour la France, ce jeune homme espère échapper à sa mobilisation à la veille d'un nouveau conflit avec l'Allemagne ; espoir vite déçu car la réponse de l'administration est aussi lapidaire que définitive : "Vous n'êtes pas pupille de la Nation "54. Quoi qu'il en soit, cet exemple montre à quel point connaître ses origines, ou, à défaut, les inventer, est une nécessité dans la construction de l'identité de ces enfants sans famille: immergés dans un milieu militaire prompt à exalter les vertus viriles, confrontés à l'imminence d'une guerre, ces garçons s'interrogent sur ce que serait leur propre conduite au front et s'inventent une filiation héroïque pour se donner du courage ou, au contraire, pour trouver une raison honorable d'échapper à l'expérience du feu.

Ces écrits soulignent l'infirmité que constitue pour les pupilles et anciens pupilles de l'Assistance publique l'ignorance de leurs origines et montrent l'étendue de la palette des stratégies déployées pour tenter d'y remédier. Si leur quête aboutit rarement, ce sont parfois leurs enfants et petits-enfants qui reprennent le flambeau, souvent avec succès ${ }^{55}$. Car cette volonté de savoir se transmet de génération en génération, dans ces familles où le secret que l'on reçoit en héritage est un silence originel, que seul le bon vouloir de l'administration pourrait rompre. Quant au rite de passage que constitue pour les pupilles cette recherche de leur filiation, il est comme suspendu, sans efficace ; à tel point que leur entrée véritable dans la vie adulte semble toujours remise à plus tard, sans cesse différée, tant qu'ils ignoreront d'où ils viennent, tant qu'il leur sera interdit de prendre cette responsabilité essentielle : choisir d'affronter la vérité de leur histoire ou continuer de l'ignorer, décider s'ils veulent tenter, à leurs risques et périls, de renouer avec une mère qui n'a pas pu ou pas voulu les garder auprès d'elle. À leur façon, ces écrits témoignent ainsi de la tragédie de ne jamais être autorisé à sortir de l'état de minorité, de ne jamais devenir un adulte à part entière.

Quant au silence de l'administration, s'il est destiné à l'origine à sauver les enfants de l'avortement ou de l'infanticide, en donnant la garantie aux femmes qu'elles peuvent abandonner leur nouveau-né sans que leur identité ne soit jamais révélée, il est, dans le même temps, revendiqué par l'Assistance publique comme la condition indispensable à la réalisation de son projet éducatif et régénérateur qui impose d'arracher définitivement les enfants à un milieu familial considéré comme corrupteur et criminogène. À la fin du $\mathrm{xx}^{\mathrm{e}}$ siècle, à mesure que s'affirme un droit d'accès aux origines, ce secret administratif trouve une nouvelle justification dans la préservation de l'harmonie des familles adoptives ${ }^{56}$ : le fait de révéler aux enfants l'identité de ceux qui les ont mis au monde risquerait de les détourner de ceux qui les ont élevés, et de troubler leur équilibre affectif et psychologique. Les termes du débat ont changé, mais, finalement, plus d'un siècle durant, c'est toujours au nom de leur propre intérêt que les enfants abandonnés se voient dénier le droit de connaître leur histoire, toute leur histoire. 


\section{NOTES}

1. De la fin du $x_{x}{ }^{e}$ siècle à l'entre-deux-guerres, les mères qui abandonnent leur enfant à l'Assistance publique de la Seine sont pour la plupart de jeunes célibataires ou des femmes séparées de leur mari. Trop pauvres pour subvenir seules aux besoins de leur nouveau-né, ou désignées à l'opprobre populaire du fait de leur situation de "filles-mères » et désireuses de cacher leur «faute » à leurs parents, ou bien sommées par ces derniers de faire disparaitre une naissance qui causerait le «déshonneur » de la famille, elles sont contraintes de se séparer de leur enfant. Certains abandons, notamment pendant la Grande guerre, concernent aussi des enfants adultérins que leur mère veut cacher à son mari. Les abandons accomplis par les pères, quelques dizaines par an, sont presque systématiquement le fait d'hommes veufs, qui, forcés de travailler en dehors de leur domicile pour un salaire très faible, ne peuvent ni s'occuper euxmêmes de leur enfant ni payer les frais d'une nourrice.

2. En particulier ceux d'Ivan Jablonka, auxquels cet article doit beaucoup. Ivan Jablonka, Ni père ni mère. Histoire des enfants de l'Assistance publique (1874-1939). Paris, Éditions du Seuil, 2006.

3. Le corpus documentaire sur lequel s'appuie cet article est constitué de 3300 dossiers de pupilles admis à l'Assistance publique parisienne entre 1904 et 1923, ce qui représente $5 \%$ de la totalité des dossiers d'enfants abandonnés sur cette période. Environ un tiers des enfants meurt avant d'être en âge d'aller à l'école.

4. Ces écrits des pupilles ne donnent sans doute qu'un aperçu limité de l'ampleur de cette quête des origines : il est très probable que certains de ces jeunes aient demandé des renseignements sur leurs parents non pas au directeur de l'Assistance publique, mais au directeur de l'agence auprès de laquelle ils étaient placés, ou à l'inspecteur du service des enfants assistés, et que ceuxci n'aient pas versé les lettres au dossier du pupille. En outre, les dossiers tenus par les agences de province, susceptibles de contenir cette correspondance locale, sont quelquefois absents des dossiers conservés à l'époque au siège parisien du service et aujourd'hui aux archives de l'aide sociale à l'enfance. Quant aux questions posées, oralement ou dans leurs lettres, par les pupilles à leurs nourriciers, elles échappent presque totalement à une investigation centrée sur les seules archives administratives.

5. Même si, en pratique, la non-communication des dossiers individuels des enfants assistés reste la règle, à la fin des années 1970, les associations d'anciens pupilles, qui se sont structurées, donnent une audience plus grande à leur revendication principale, la levée du secret de la filiation. Dans le même temps, l'évolution des mœurs administratives, avec, par exemple, la création par la loi du 17 juillet 1978 de la Commission d'accès aux documents administratifs (CADA), va dans le sens d'une amélioration de l'accès des citoyens aux informations personnelles que détiennent les administrations publiques. La loi du 11 juillet 1979 étend le principe de la liberté d'accès aux informations personnelles aux dossiers individuels, sauf dans le cas de «secrets protégés par la loi »; ce qui exclut les renseignements relatifs à la filiation des enfants abandonnés, mais permet aux services de l'aide à l'enfance, tout en taisant ce qui pourrait révéler l'identité des parents, de faire connaître aux anciens pupilles les circonstances et les motifs de leur abandon.

6. Il s'agit des 72 pupilles qui, parmi les 454 enfants abandonnés au cours du premier trimestre 1923, ont survécu à la très forte mortalité de la petite enfance, et ont, par la suite, écrit à l'administration afin d'obtenir des renseignements sur leurs parents et sur les raisons de leur abandon.

7. Sont comptés parmi ces démarches couronnées de succès tous les cas où l'administration communique des renseignements, même partiels (il est fréquent, par exemple, que la copie du dossier communiquée à l'ancien pupille soit expurgée des informations relatives à l'identité des 
parents, ou que l'administration écrive au demandeur une lettre dans laquelle les circonstances de l'abandon sont résumées en une phrase et où rien n'est dit qui pourrait permettre de retrouver la famille maternelle).

8. Les différentes catégories d'enfants assistés sont fixées par la loi du 27 juin 1904.

9. La pratique des expositions d'enfant dans un lieu public est largement tombée en désuétude depuis le milieu du $\mathrm{XIX}^{\mathrm{e}}$ siècle, et l'Assistance publique parisienne ne recueille plus que quelques dizaines d'enfants exposés par an.

10. L'expression « la personne qui dépose l'enfant refuse de se faire connaître » est couramment utilisée dans les procès-verbaux d'admission des enfants immatriculés dans la catégorie des «trouvés».

11. En réalité, l'administration connaît le nom de la mère de quelques "trouvés », parfois aussi les circonstances de leur abandon; il s'agit de pupilles dont la mère, sans doute dans l'espoir de reprendre un jour son enfant, a indiqué son identité et accepté de répondre à quelques questions lors du dépôt à l'hospice, mais dont la filiation n'est pas officiellement établie, puisqu'ils ont été déclarés à l'état civil comme " nés de père et de mère non dénommés ".

12. Paul Strauss (1852-1942), conseiller municipal de Paris (1883) et rapporteur du service des enfants assistés au Conseil général de la Seine, puis sénateur de la Seine (1897), est l'un des principaux artisans de la loi du 27 juin 1904 sur le service des enfants assistés. Son nom reste aussi attaché à la loi du 17 juin 1913 sur le repos des femmes en couches. Bénéficiant d'un impressionnant réseau dans les milieux philanthropiques, médicaux, juridiques et politiques, il est tout au long de la Troisième République au cœur de la «nébuleuse réformatrice » de l'assistance maternelle et infantile (l'expression est de Christian Topalov, Laboratoires du nouveau siècle. La nébuleuse réformatrice et ses réseaux en France, 1880-1914, Paris, Éditions de l'EHESS, 1999). Sommet de sa carrière, Paul Strauss est, de janvier 1922 à mars 1924, ministre de l'Hygiène, de l'Assistance et de la prévoyance sociales.

13. L'expression de Paul Strauss est citée par Sébastien Turquan dans : «Le tour et le bureau ouvert ", La Revue philanthropique, t. 40, avril 1919, p.137-151. Cet article est une synthèse du rapport présenté par l'auteur au Conseil supérieur de l'Assistance Publique dans sa séance du 26 février 1919.

14. Ivan Jablonka, op. cit.

15. Conseil supérieur de l'Assistance publique, « Rapport sur le projet de loi relatif au service des enfants assistés, présenté par M. Brueyre », 1889, in Sénat, Documents parlementaires, annexe $n^{\circ} 27$, séance du 18 février 1892, p. 116.

16. «Rapport sur le projet de loi relatif au service des enfants assistés, présenté par M. Théophile Roussel ", 1892 in Sénat, Documents parlementaires, annexe $n^{\circ} 27$, séance du 18 février 1892, p. 77.

17. Dans cette lettre datée de juillet 1981, l'ancien pupille renouvelle une démarche qu'il a effectuée au moins trois fois depuis 1940, et demande des renseignements sur sa mère, même s'il pense qu'il n'y a plus d'espoir de la retrouver vivante : il se présente comme " un vieil homme » et affirme que combler « ce besoin de [ses] origines [...] [qui lui] tient à l'âme et au corps depuis [son] enfance » serait sa " dernière grande joie ». Direction de l'action sociale, de l'enfance et de la santé de la ville de Paris ( DASES), dossier d'un enfant admis au service des enfants assistés de la Seine en février 1918.

18. Lettre de juillet 1981, DASES, dossier d'un enfant admis au service des enfants assistés de la Seine en février 1918.

19. L'analyse ne fait ici que confirmer les conclusions d'Ivan Jablonka: «On pourrait croire que ces interrogations sont suscitées par la solitude, la détresse ou la gêne; mais le souci de la parenté biologique s'observe aussi chez les pupilles qui, quasiment adoptés par leurs nourriciers, ont eu une enfance stable et heureuse. » Ivan Jablonka, op. cit., p. 258. 
20. L'un d'eux, alors qu'il va fêter ses 21 ans, écrit en novembre 1933 au directeur de l'administration, afin de lui demander des renseignements sur sa mère; il commence sa lettre par ces mots : "Je sais que vous allez répondre négativement à ma demande ", témoignant de toute l'ambiguïté du rapport que ces jeunes entretiennent avec une règle du secret qu'ils ont intégrée mais qu'ils espèrent pouvoir transgresser. DASES, dossier d'un enfant admis au service des enfants assistés de la Seine en janvier 1913.

21. Il semble que quelques parents abandonneurs partagent cette croyance et redoutent qu'à la majorité des pupilles leur filiation ne leur soit révélée. C'est le cas de cette femme, qui en 1919 a fait l'abandon du petit garçon qu'elle a eu à la suite d'un viol commis par un soldat allemand, lorsqu'elle résidait en territoire envahi ; en 1938, elle écrit au directeur de l'Assistance publique de Paris : « comme dans ma détresse j'ai donné mon nom [lors de l'abandon] en réclamant bien le secret, j'ai donc l'honneur, Monsieur le Directeur, de venir vous demander l'obligeance, au cas où mon garçon quand il aura 20 ans il vous demanderait des renseignements, de vouloir garder ce secret. » DASES, dossier d'un enfant admis au service des enfants assistés de la Seine en mai 1917.

22. DASES, dossier d'un enfant admis au service des enfants assistés de la Seine en avril 1917.

23. DASES, dossier d'un enfant admis au service des enfants assistés de la Seine en juin 1917.

24. Souligné par nous.

25. Aucune dérogation n'est finalement accordée à ces pupilles mobilisés, car, poursuit le rapport, «le secret professionnel ne permet pas de leur donner satisfaction». Administration générale de l'Assistance publique à Paris, Rapport sur le service des enfants assistés du département de la Seine pendant l'année 1918, Paris, 1919, p. 26.

26. Les rapports entre l'Assistance publique et les familles nourricières sont aussi organisés autour d'un système de récompense : "primes de survie », lorsque les nourrissons atteignent sans encombre leur deuxième année, "primes de fréquentation scolaire", "primes exceptionnelles ", lorsqu'elles ont fait preuve d'un dévouement exemplaire, les incitent à bien s'occuper des enfants.

27. Ivan JABLONKA, op. cit., p. 59.

28. Lettre d'une pupille de 20 ans au directeur de l'Assistance publique, datée de mai 1924 . DASES, dossier d'un enfant admis au service des enfants assistés de la Seine en février 1904.

29. Les «mises en relations" se distinguent des "remises» d'enfants par le fait qu'elles ne donnent pas lieu à une restitution de la tutelle aux parents.

30. La persévérance des parents est aussi mise à l'épreuve, puisqu'il est rare qu'une remise ou une mise en relation soit accordée dès la première demande.

31. Environ $6 \%$ des enfants admis au cours du premier trimestre de 1923 sont remis à leurs parents, et $2 \%$ font l'objet d'une «mise en relations "; les proportions sont respectivement de $4 \%$ et de $1 \%$ pour la période de la Grande Guerre.

32. DASES, dossier d'un enfant admis au service des enfants assistés de la Seine en mars 1916.

33. Par exemple, la découverte, en 1900 , des groupes sanguins et des règles héréditaires qui les régissent suscite dans l'entre-deux-guerres un vif intérêt, parfois dévoyé par ceux qui font du sang le support des identités communautaires, familiales, nationales ou raciales. C'est en 1924, en Allemagne, que les connaissances scientifiques sur les groupes sanguins sont utilisées pour la première fois dans une affaire judiciaire de déclaration de paternité ; en France, il faut attendre 1937. Voir : Louis Christiaens, La Recherche de la paternité par les groupes sanguins. Étude technique et juridique, Paris, Masson, 1939.

34. Finalement le mariage a lieu, aux États-Unis, mais seulement quatre ans plus tard, en 1951. DASES, dossier d'un enfant admis au service des enfants assistés de la Seine en janvier 1923.

35. Expression citée plus haut. DASES, dossier d'un enfant admis au service des enfants assistés de la Seine en février 1918.

36. DASES, dossier d'un enfant admis au service des enfants assistés de la Seine en juin 1917. 
37. DASES, dossier d'un enfant admis au service des enfants assistés de la Seine en septembre 1919.

38. Lettre, déjà citée, datée de juillet 1981. DASES, dossier d'un enfant admis au service des enfants assistés de la Seine en février 1918.

39. Lettre datée de décembre 1990. DASES, dossier d'un enfant admis au service des enfants assistés de la Seine en juillet 1919.

40. Bien que, sans doute, ces retrouvailles clandestines échappent pour la plupart à l'administration, les archives de l'Assistance publique en donnent quelques exemples : un ancien pupille, abandonné à l'âge de 2 ans en janvier 1918, retrouve sa mère en 1961 grâce à l'aide du juge des enfants de Beauvais dont son épouse est la greffière (DASES, dossier d'un enfant admis au service des enfants assistés de la Seine en janvier 1918); une femme de 38 ans retrouve en 1954 sa sœur aînée, qui n'avait pas été abandonnée et était demeurée avec leurs parents, grâce à une annonce que celle-ci a fait paraître dans les journaux (DASES, dossier d'un enfant admis au service des enfants assistés de la Seine en janvier 1918).

41. Depuis la loi du 28 mars 1882 l'école est obligatoire pour les enfants de 6 à 13 ans.

42. Un pupille, né en 1904 et abandonné à la naissance dans la catégorie des trouvés, a été pourvu, du fait d'une négligence de l'administration, d'un état civil constitué seulement d'un prénom. En 1932, il désire acheter une maison pour y vivre avec sa femme, mais le notaire n'accepte ni l'acte d'état civil sans nom patronymique, ni le certificat d'origine que lui fournit l'ancien pupille, et il faut l'intervention de l'Assistance publique pour que la vente ait finalement lieu, au bout de plusieurs mois de démarches administratives. DASES, dossier d'un enfant admis au service des enfants assistés de la Seine en février 1904.

43. DASES, dossier d'un enfant admis au service des enfants assistés de la Seine en février 1919.

44. L'Assistance publique semble obsédée par la crainte que ses garçons ne deviennent des voleurs ou des escrocs et que ses filles ne se prostituent; cela tient à ce que l'une des justifications de son intervention auprès des enfants assistés est justement d'éviter qu'ils ne tournent mal. Dans les travaux préparatoires de la loi du 24 juillet 1889 sur les enfants moralement abandonnés, le principe de la déchéance de la puissance paternelle à l'encontre de parents «indignes» est ainsi justifié, entre autres, par la nécessité d'éviter que les enfants concernés ne deviennent des criminels. Le Conseil supérieur de l'Assistance publique affirme, par exemple, à propos des dispositions de cette loi : " elles transformeront en honnêtes citoyens, en braves mères de familles des enfants qui, si la société ne leur tend pas la main, deviendront un jour ses pires ennemis et peupleront ses prisons, ses bagnes, ses lupanars. " «Rapport sur le projet de loi relatif à la protection des enfants maltraités ou moralement abandonnés, présenté par M. Brueyre ", 1888, Documents parlementaires. Chambre des députés, annexe n³389, séance du 22 décembre 1888, p. 728.

45. DASES, dossier d'un enfant admis au service des enfants assistés de la Seine en mars 1916.

46. DASES, dossier d'un enfant admis au service des enfants assistés de la Seine en décembre 1922.

47. DASES, dossier d'un enfant admis au service des enfants assistés de la Seine en mars 1918.

48. DASES, dossier d'un enfant admis au service des enfants assistés de la Seine en juillet 1917.

49. Ivan Jablonka, op. cit., p. 260.

50. DASES, dossier d'un enfant admis au service des enfants assistés de la Seine en février 1923.

51. DASES, dossier d'un enfant admis au service des enfants assistés de la Seine en août 1917.

52. DASES, dossier d'un enfant admis au service des enfants assistés de la Seine en mars 1916.

53. DASES, dossier d'un enfant admis au service des enfants assistés de la Seine en mai 1919.

54. DASES, dossier d'un enfant admis au service des enfants assistés de la Seine en mai 1919.

55. L'accès des descendants des pupilles aux dossiers individuels est facilité par une plus grande prise en compte, depuis la fin du $\mathrm{xx}^{\mathrm{e}}$ siècle, de la revendication des enfants nés sous $\mathrm{X}$ de connaître l'identité de leurs parents, ainsi que par une certaine sensibilisation de l'opinion 
publique au thème du « droit d'accès aux origines ». La dernière avancée en la matière est opérée par la loi du 10 janvier 2002, dont l'objectif est de substituer à l'accouchement "anonyme » l'accouchement "dans le secret", et d'organiser la réversibilité du secret à la demande de l'enfant après accord du parent de naissance concerné. La loi crée le Conseil national pour l'accès aux origines personnelles (CNAOP), qui est saisi des demandes des anciens pupilles, qui les instruit, puis contacte confidentiellement les mères de naissance, et, enfin, organise, le cas échéant, les modalités pratiques de la levée du secret, de la consultation du dossier, voire de l'échange de correspondance entre les personnes concernées.

56. Avant la loi du 19 juin 1923 l'adoption des mineurs n'est pas possible en droit français. C'est la loi du 29 juillet 1939 sur la légitimation adoptive qui crée le principe d'une filiation adoptive irrévocable. Enfin, la loi du 11 juillet 1966 crée l'adoption plénière. Sur l'adoption, voir : Agnès Fine et Claire Neirinck (dir.), Parents de sang, Parents adoptifs, Paris, Librairie générale de droit et de jurisprudence, 2000 ; et : Nadine Lefaucheur, «Accouchement sous X et mères de l'ombre ", in Didier Le Gall et Yamina Bettahar (dir.), Pluriparentalités, Paris, PUF, 2000.

\section{RÉSUMÉS}

Au début de la Troisième République, l'Assistance publique de Paris recueille chaque année environ 3000 enfants abandonnés. Séparés d'une famille dont ils ne gardent aucun souvenir, ces jeunes grandissent dans l'ignorance de leurs origines, conformément à la loi et à la culture administrative de l'institution. L'identité de leurs parents, les motifs et les circonstances de leur abandon sont pourtant des énigmes qui hantent la plupart d'entre eux, et dont ils imaginent que l'administration détient toutes les clés. Leur correspondance avec le directeur de l'Assistance publique témoigne de cette douloureuse obsession, et laisse apparaitre le roman que ces enfants sans famille se sont parfois inventé pour combler les silences de leur histoire personnelle. Elle révèle aussi la diversité des stratégies épistolaires mises en place pour infléchir la règle du secret, ainsi que les circonstances de cette prise d'écriture qui rythme les étapes importantes de leur vie de jeunes adultes : service militaire, majorité, mariage ou naissance de leur premier enfant.

\section{The Search for origins}

Children of the French Assistance Publique in search of their personal history (1900-1920) At the beginning of the Third Republic, services of the Paris Assistance Publique collected about 3000 abandoned children a year from the streets. Separated from a family of which they had no memory, these children grew up ignorant of their origins, in accordance with the law and the administrative culture of the institution. The identity of their parents and the motives and circumstances of their abandonment were enigmas plaguing most of them, convinced as they were that the administration knew where they came from. Their correspondence with the Director of the Assistance Publique bears evidence of this painful obsession. It discloses the fiction these children without families made up to fill in the gaps of their biographies. It also reveals the various epistolary strategies implemented to bypass the confidentiality rules and the circumstances of their adulthood in which they penned their milestones - entering the army, reaching majority, getting married or having their first child. 
INDEX

Index géographique : France

Index chronologique : Entre deux guerres

Mots-clés : assistance publique, dossier administratif, filiation, origine, roman familial, stratégies épistolaires

\section{AUTEUR}

ANTOINE RIVIÈRE

Doctorant à l'université de Paris IV 